Cutts, N. S. \& Rainbow, C. (1951). J. gen. Microbiol. 5, 860-868.

\title{
Non-competitive Sulphanilamide Antagonists for Yeasts
}

\author{
By N. S. CUTTS AND C. RAINBOW \\ Department of Industrial Fermentation, University of Birmingham
}

SUMMARY: Sulphanilamide inhibition of the growth of certain yeasts and yeastlike organisms was overcome in a non-competitive manner by methionine, adenine and histidine, which are therefore regarded as end-products of enzyme systems for which $p$-aminobenzoic acid is essential. The methionine system was the most sensitive to sulphanilamide, followed in order by those of adenine and histidine.

In the presence of methionine, adenine and histidine together, the test organisms were still sensitive to sulphanilamide, implying that $p$-aminobenzoic acid is concerned in still another system, or systems. The nature of these systems is obscure, but the inhibition is not overcome by purines, pyrimidines or pteroylglutamic acid, nor by single amino-acids, except in one case when DL-cysteine showed some effect. In another case, a low concentration of choline chloride was effective.

Evidence of the vital functions of $p$-aminobenzoic acid ( $p$-AB) may be obtained by the method of inhibition analysis using sulphanilamide (S-amide) (Shive \& Roberts, 1946). The biosynthesis of methionine and of the purine skeleton seems to be dependent on enzyme systems in which $p$-AB plays an essential role, since methionine and purines overcome S-amide inhibition in a noncompetitive manner (Harris \& Kohn, 1941; Snell \& Mitchell, 1942; Lampen \& Jones, 1947). This conclusion is supported by other studies which showed that $p$-AB can be replaced by purines and amino-acids in the growth of mutant strains of Escherichia coli (Lampen, Jones \& Roepke, 1949) and by adenine, methionine and histidine for the growth of a yeast (Cutts \& Rainbow, 1950).

In other cases, $\mathrm{S}$-amide inhibition is overcome by relatively high concentrations of thymine or by relatively low concentrations of pteroylglutamic acid (PGA). It is therefore concluded that PGA is concerned in the biosynthesis of thymine (Lampen \& Jones, 1947; Winkler \& de Haan, 1948). As a result of extensive inhibition analysis of Esch. coli, the latter workers found that serine is an S-amide antagonist, and Lampen et al. (1949) showed that other aminoacids have some effect. In the present study, purines, pyrimidines, aminoacids, PGA and choline were tested as antagonists of S-amide bacteriostasis of some yeast and yeast-like organisms, and the results compared with those already obtained by a different technique (Cutts \& Rainbow, 1950).

\section{METHODS}

The basal medium used was that described by Cutts \& Rainbow (1950).

Test organisms and inocula. Four test organisms were used; cultures of each were maintained on malt agar slants and transferred 2-3 times weekly as required.

(a) The strain of Saccharomyces cerevisiae, designated ' Yeast 47' by Rainbow (1948). This organism requires exogenous sources of biotin, pantothenate and 
$p$-AB. Inocula were prepared by transferring a little surface growth from a malt agar slant to $6 \mathrm{ml}$. of basal medium containing $0 \cdot 1 \mu \mathrm{g} . p-\mathrm{AB} / \mathrm{ml}$. and incubating at $25^{\circ}$ for $18-24 \mathrm{hr}$. The cells were then washed twice (centrifuge) in $6 \mathrm{ml}$. portions of sterile $0.85 \%$ saline and finally suspended in a further $6 \mathrm{ml}$. portion.

(b) A strain of top fermentation brewer's yeast ( $S$. cerevisiae) isolated by us from a brewery pitching yeast and designated 'Yeast 70'. This strain required biotin and inositol, synthesized pantothenate slowly, but was independent of exogenous $p$-AB. Inocula were suspensions in sterile saline of a little of the surface growth from a malt agar slant.

(c) Kloeckera brevis, B768 (Emery, McLeod \& Robinson, 1946). This strain was independent of exogenous $p$-AB. Inocula were prepared as for $(b)$ above.

(d) Schizosaccharomyces octosporus, an organism requiring for growth exogenous adenine, methionine and histidine, but not $p$-AB (Northam \& Norris, 1951). Inocula were grown in basal medium supplemented with ( $\mu \mathrm{g} . / \mathrm{ml}$.$) : DL-methionine (100), adenine \mathrm{HCl}(50)$ and L-histidine $\mathrm{HCl}(25)$ and otherwise treated as in $(a)$ above.

Auxanographic tests were carried out as previously described (Cutts \& Rainbow, 1950). For each series, the following substances were tested for ability to overcome sulphanilamide inhibition: purines and pyrimidines-adenine, guanine, xanthine, cytosine, thymine and uracil; amino-acids-DL-alanine, L-arginine, L-asparagine, L-aspartic acid, DL-cysteine, L-cystine, glycine, L-glutamic acid, L-histidine, L-hydroxyproline, DL-isoleucine, L-leucine, L-lysine, DL-methionine, DL-norleucine, DL-norvaline, L-proline, DL-phenylalanine, DL-serine, DL-threonine, DL-tryptophan, L-tyrosine and DL-valine; other substances- $p$-AB, PGA and choline chloride.

Tests in liquid media were carried out as described by Northam \& Norris (1951). Observations were made on duplicate cultures.

\section{RESULTS}

\section{Inhibition of Yeast 47}

Auxanographic tests were carried out to identify substances which could overcome the inhibition of the growth of Yeast 47 by sulphanilamide (S-amide). The substances listed above were first tested in the basal medium containing $1000 \mu \mathrm{g}$. S-amide and $0.5 \mu \mathrm{g}$. $p$-AB $/ \mathrm{ml}$. In this medium, little growth took place after $48 \mathrm{hr}$. incubation, during which period observations were made. After $96 \mathrm{hr}$. growth was heavy and general on all plates, except around the point of application of norleucine. The results are recorded in Table 1. Apart from $p$-AB, DL-methionine, adenine, guanine and DL-cysteine each overcame S-amide inhibition, whilst norleucine accentuated the inhibition.

For the second series of auxanographs, DL-methionine adenine and guanine were added singly to portions of basal medium containing a concentration of $p$-AB decreased to permit inhibition of growth by $1000 \mu \mathrm{g}$. S-amide/ml. In these tests, S-amide inhibition was overcome by adenine or by guanine in the medium containing methionine, or by DL-methionine in the media containing 
adenine or guanine. Norleucine was again inhibitory in the two latter media.

In basal medium containing S-amide, $p$-AB, DL-methionine and adenine, inhibition of Yeast 47 was overcome only by L-histidine and by $p$-AB itself. $\mathrm{By}$ including $\mathrm{L}$-histidine $\mathrm{HCl}$ in the basal medium, as well as adenine and methionine, and diminishing the $p$-AB concentration still further to permit of inhibition, Yeast 47 was found too tolerant of S-amide for further results to be obtained, unless the inoculum used for the tests was one.of deficient cells obtained by growth in the defined $p$-AB-free medium described in $(d)$ above. In this way, DL-cysteine was shown to overcome S-amide inhibition in the presence of methionine, adenine and histidine.

For the final auxanographs, adenine, methionine, histidine and DL-cysteine were included in the basal medium containing $\mathrm{S}$-amide and $p$-AB. Under these conditions and using an inoculum from the $p$-AB free defined medium,

\section{Table 1. Antagonists of sulphanilamide inhibition of various organisms}

(Results were obtained by auxanograph technique; observations up to $48 \mathrm{hr}$. of incubation at $25^{\circ}$.)

Basal medium containing

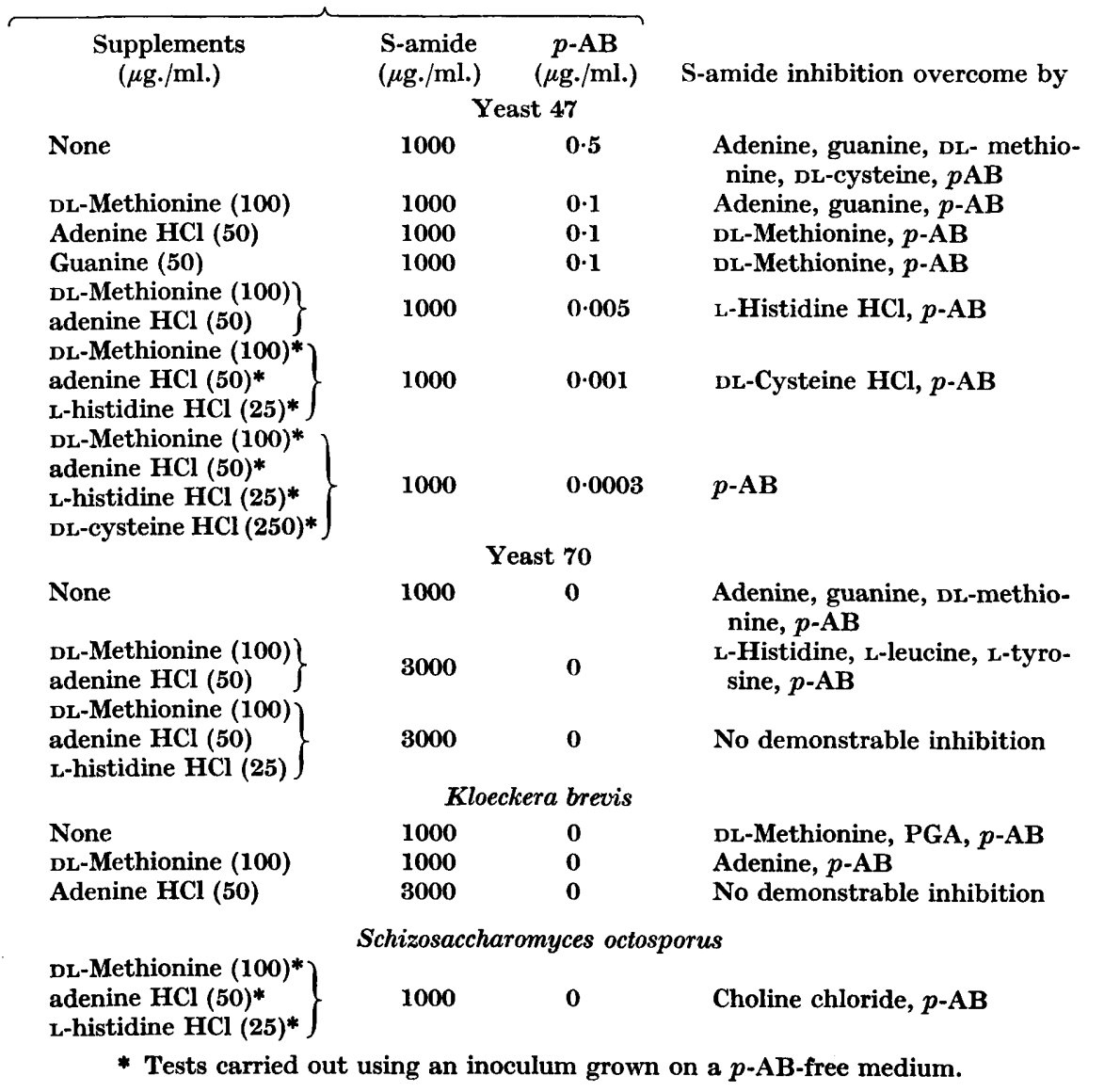


Yeast 47 was still sensitive to S-amide, the inhibition being overcome only by $p$-AB of all the substances tested.

Non-competitive nature of the anti-S-amide action of purines and amino-acids. For Yeast 47, a competitive relation was found to exist between $p$-AB and concentrations of S-amide between 300 and $3000 \mu \mathrm{g} . / \mathrm{ml}$. For lower concentrations of $\mathrm{S}$-amide, the ratio of the concentrations (S-amide) $/(p-\mathrm{AB})$ required just to restore full growth (i.e. the antibacterial index) diminished, as might be expected when the enzyme systems involved are no longer saturated with respect to $p$-AB and $\mathrm{S}$-amide. At the other extreme, the inhibition due to $10,000 \mu \mathrm{g}$. S-amide/ml. was not completely overcome by $p$-AB.

The non-competitive nature of the action of methionine, adenine and histidine in overcoming the S-amide inhibition of Yeast 47 was demonstrated in liquid medium containing $1000 \mu \mathrm{g}$. S-amide/ml. Fig. 1 shows that methionine behaved as a non-competitive antagonist. The concentration of $p$-AB required to restore full growth was decreased from $1 \cdot 0$ to $0 \cdot 3 \mu \mathrm{g} . p-\mathrm{AB} / \mathrm{ml}$. (approximately a threefold increase in the antibacterial index) by $100 \mu$ g. of DL-methionine/ml. This concentration of methionine partially overcame the inhibition in the presence of $0 \cdot 1 \mu \mathrm{g} . p-\mathrm{AB} / \mathrm{ml}$., but higher methionine concentrations, which would be expected to be more effective if the relation were competitive, were no more effective.

The non-competitive anti-S-amide activities of adenine and histidine are shown in Figs. 2 and 3 respectively. The effect of adenine was evident in a medium containing $100 \mu \mathrm{g}$. DL-methionine $/ \mathrm{ml}$., $30 \mu \mathrm{g}$. adenine $\mathrm{HCl} / \mathrm{ml}$. producing a further threefold increase in the antibacterial index. In a separate experiment, guanine was found to be equally effective. To demonstrate the non-competitive anti-S-amide activity of histidine, the presence in the medium of both DL-methionine $(100 \mu \mathrm{g} . / \mathrm{ml}$. $)$ and adenine $\mathrm{HCl}(100 \mu \mathrm{g} . / \mathrm{ml}$. $)$ was necessary. In this case, the inhibition in $p$-AB-free medium was partially overcome by $100 \mu \mathrm{g}$. L-histidine $\mathrm{HCl} / \mathrm{ml}$; $1000 \mu \mathrm{g}$. L-histidine/ml. was no more effective. Thus for Yeast 47 methionine, adenine and histidine are non-competitive S-amide antagonists, which suggests that these are end-products of enzymic systems for which $p$-AB is essential.

The order in which methionine, adenine and histidine exert antisulphonamide activity. The effects on the antibacterial index of additions of methionine, adenine and histidine, singly and in all possible combinations, to the basal medium was determined by tube tests (Table 2). The antibacterial index was almost unaltered by additions of adenine or histidine, or by adenine + histidine, but was increased some 2.5 times by methionine. The effect of histidine and methionine together was no greater than that of methionine alone, but the combination adenine + methione resulted in a further $2 \cdot 5$-fold increase of the index. The effect of histidine was evident only when adenine and methionine are both present.

It therefore appears that the anti-S-amide actions of these substances are exerted in a definite order, the methionine system being the most sensitive to S-amide, that of adenine intermediate and that of histidine the least sensitive. The successive effects of the three substances is shown in Fig. 4. 


\section{Inhibitions of other yeasts}

To examine whether the results obtained with Yeast 47 were of more general significance, other yeasts and yeast-like organisms were tested. Only qualitative tests were made, by means of the auxanographic technique, with the minor

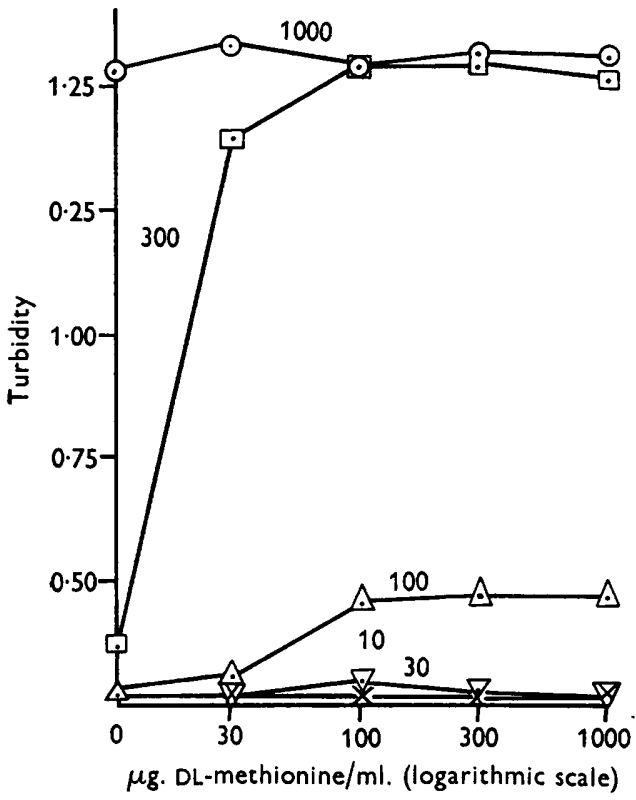

Fig. 1

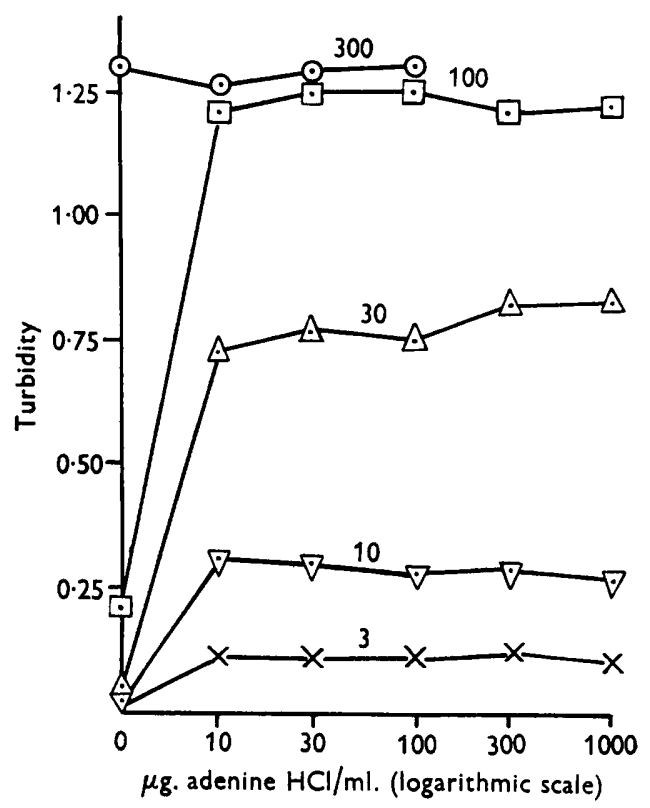

Fig. 2

Fig. 1. S-amide inhibition (1000 $\mu \mathrm{g}$. S-amide/ml.) of Yeast 47 overcome by DL-methionine. The numbers above the curves show the respective concentrations of $p-A B(m \mu g . / m l$.$) .$

Fig. 2. Inhibition of Yeast 47 in presence of $1000 \mu \mathrm{g}$. S-amide $+100 \mu \mathrm{g}$. DL-methionine overcome by adenine at various concentrations of $p-\mathrm{AB}(p-\mathrm{AB}$ in $\mathrm{m} \mu \mathrm{g} . / \mathrm{ml}$. as shown by the numbers above the respective curves).

Table 2. Effects of methionine, adenine and histidine on the antibacterial index with Yeast 47

(Antibacterial index $=1000 / \mu \mathrm{g} \cdot p-\mathrm{AB} / \mathrm{ml}$. required just to restore full growth in $48 \mathrm{hr}$. at $25^{\circ}$. Basal medium contained $1000 \mu \mathrm{g}$. S-amide $/ \mathrm{ml}$.)

\begin{tabular}{|c|c|}
\hline $\begin{array}{l}\text { Supplements } \\
(\mu \mathrm{g} . / \mathrm{ml} .)\end{array}$ & $\begin{array}{l}\text { Antibacterial } \\
\text { index }\left(10^{3}\right)\end{array}$ \\
\hline Nil & $1 \cdot 0$ \\
\hline Adenine $\mathrm{HCl}(50)$ & $1 \cdot 0$ \\
\hline L-Histidine $\mathrm{HCl}(25)$ & $1 \cdot 0$ \\
\hline Adenine $\mathrm{HCl}(5)+$ & \\
\hline L-histidine HCl (25) & $1 \cdot 0$ \\
\hline DL-Methionine (100) & $2 \cdot 5$ \\
\hline DL-Methionine $(100)+$ & \\
\hline L-histidine $\mathrm{HCl}(\mathbf{2 5})$ & $\mathbf{2 \cdot 5}$ \\
\hline $\begin{array}{l}\text { DL-Methionine }(\mathbf{1 0 0 )}+ \\
\text { adenine } \mathrm{HCl}(\mathbf{5 0})\end{array}$ & 6.0 \\
\hline $\begin{array}{l}\text { DL-Methionine }(100)+\text { adenine } \\
\text { HCl }(50)+\text { L-histidine HCI (25) }\end{array}$ & $60 \cdot 0$ \\
\hline
\end{tabular}


modification that, since none of the organisms tested required a source of exogenous $p$-AB, this substance was omitted from the basal medium, in order that the sensitivity to $\mathrm{S}$-amide should not be diminished. The results are recorded in Table 1.

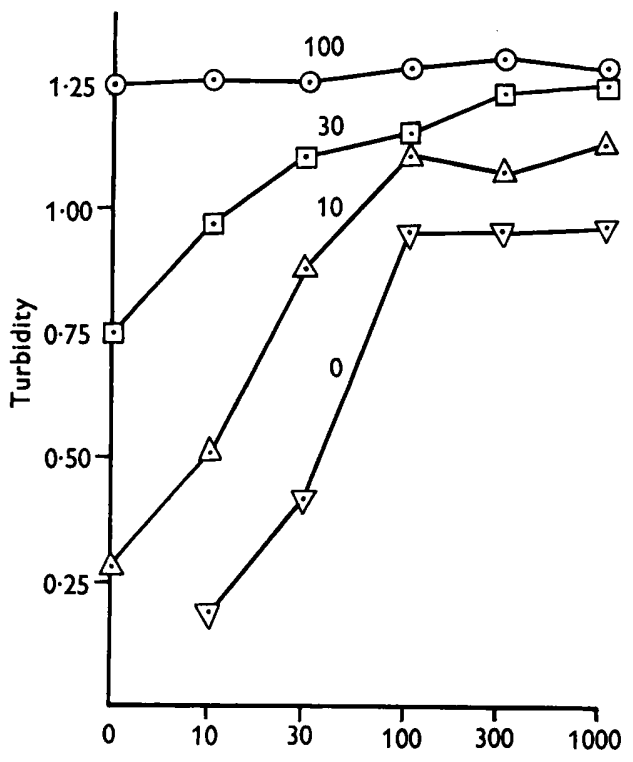

$\mu g$. L-histidine $\mathrm{HCl} / \mathrm{ml}$. (logarithmic scale)

Fig. 3

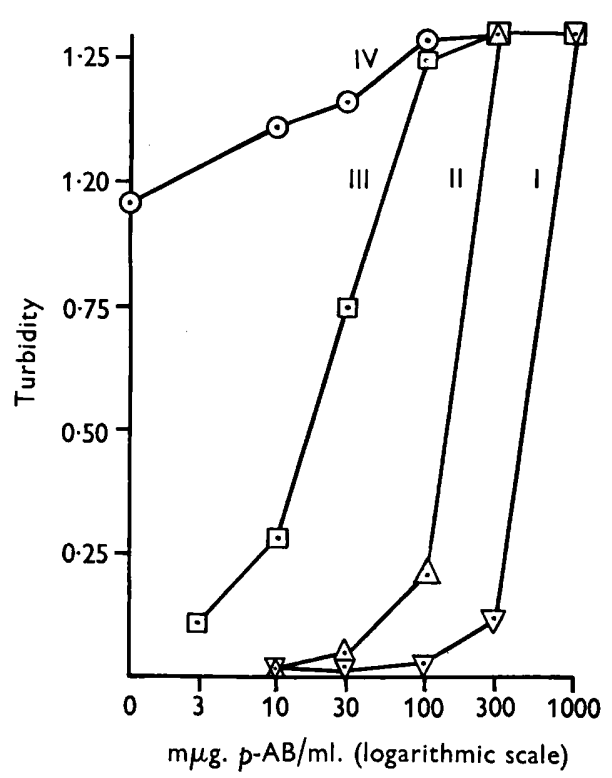

Fig. 4

Fig. 3. Inhibition of Yeast 47 in presence of S-amide $(1000 \mu \mathrm{g} . / \mathrm{ml})+$. DL-methionine $(100 \mu \mathrm{g} . / \mathrm{ml})+$. adenine $\mathrm{HCl}(100 \mu \mathrm{g} . / \mathrm{ml}$.) overcome by L-histidine at various concentrations of $p$-AB. $p$-AB concentrations are indicated by the numbers $(\mathrm{m} \mu \mathrm{g} . / \mathrm{ml}$.) above the respective curves.

Fig. 4. Inhibition of growth of Yeast 47 in basal medium $+1000 \mu \mathrm{g}$. S-amide $/ \mathrm{ml}$. overcome by $p$-AB under various conditions. Curve I, no additions; curve II, $+100 \mu \mathrm{g}$. DL-methionine $/ \mathrm{ml}$; ; curve III, $+100 \mu \mathrm{g}$. each of $\mathrm{DL}$-methionine and adenine $\mathrm{HCl} / \mathrm{ml}$. ; curve IV, $+100 \mu \mathrm{g}$. each of $\mathrm{DL}$-methionine, adenine $\mathrm{HCl}$ and L-histidine $\mathrm{HCl} / \mathrm{ml}$.

S-amide inhibition of Yeast 70 resembled that of Yeast 47 with respect to methionine, adenine and histidine, except that inhibition in the presence of methionine and adenine was overcome by L-leucine and L-tyrosine, as well as by L-histidine. Cutts $\&$ Rainbow (1950) showed that the $p$-AB-sparing effect of suboptimal concentrations of methionine was much enhanced by leucine and tyrosine, and in the present case these amino-acids may act by virtue of their methionine-sparing effect. This conclusion is supported by the fact that, on raising the concentration of DL-methionine to $250 \mu \mathrm{g} . / \mathrm{ml}$. and repeating the test with Yeast 70, no anti-S-amide activity by L-leucine or L-tyrosine could be detected.

With Kloeckera brevis, S-amide inhibition was overcome by methionine and adenine; but in the presence of both these substances, the organism was too 
tolerant of S-amide to permit of further inhibition analysis. The action of PGA as an anti-S-amide substance was approximately competitive over a limited range, a relatively high concentration $(3 \mu \mathrm{g} . / \mathrm{ml}$.$) being required to$ overcome the inhibition by $1000 \mu \mathrm{g}$. S-amide $/ \mathrm{ml}$. This suggested that the activity of the PGA was due to its conversion by $K$. brevis into free $p$-AB (cf. Koft, Sevag \& Steers, 1950).

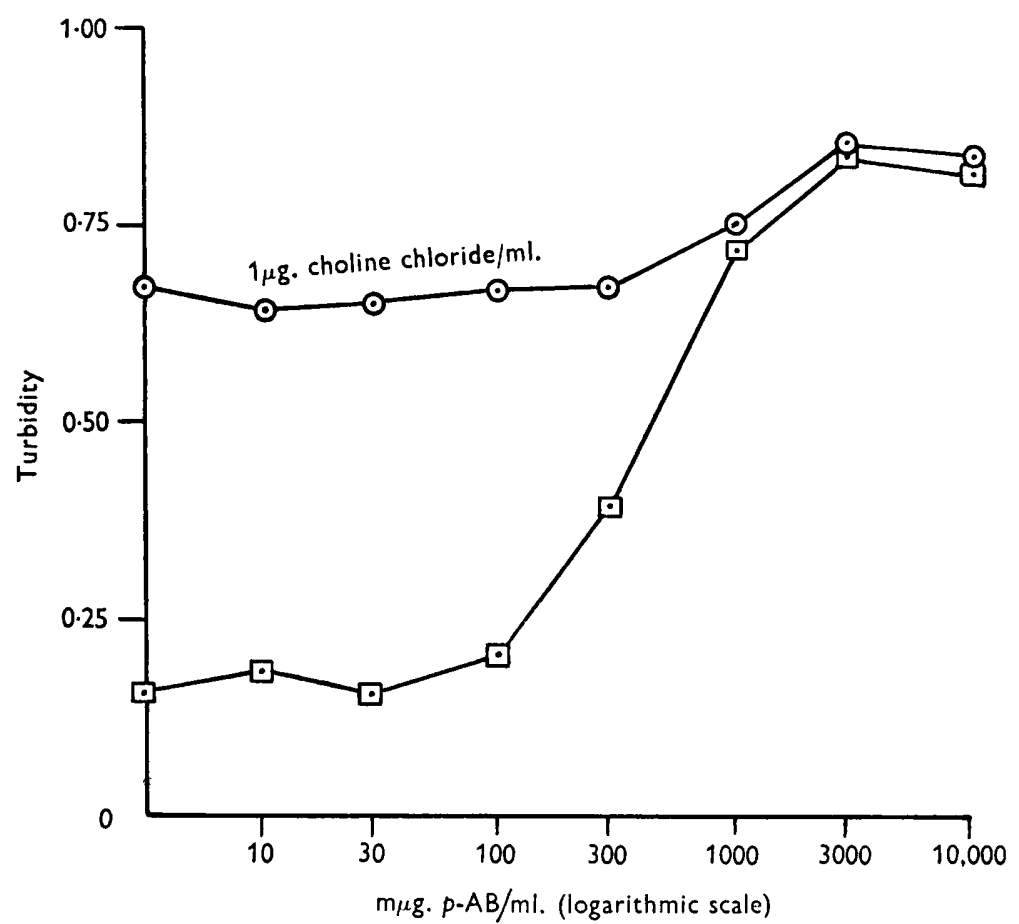

Fig. 5. Inhibition of growth of Schizosaccharomyces octosporus in basal medium $+1000 \mu \mathrm{g}$. S-amide $/ \mathrm{ml} .+100 \mu \mathrm{g}$. DL-methionine $/ \mathrm{ml} .+50 \mu \mathrm{g}$. adenine $\mathrm{HCl} / \mathrm{ml} .+25 \mu \mathrm{g}$. L-histidine $\mathrm{HCl} / \mathrm{ml}$. Inhibition overcome by $p$-AB in the presence and absence of $1 \mu \mathrm{g}$. choline chloride/ml.

Schizosaccharomyces octosporus did not require exogenous $p$-AB for growth, but had to be provided with adenine, methionine and histidine, the syntheses of which compounds could not therefore be blocked by S-amide. Nevertheless, the organism showed inhibition by S-amide, which was overcome by $1 \mu \mathrm{g}$. choline chloride/ml. (Fig. 5), although the growth rate was then subnormal; concentrations of choline chloride exceeding $1 \mu \mathrm{g} . / \mathrm{ml}$. had no greater effect.

\section{DISCUSSION}

As non-competitive antagonists of $\mathrm{S}$-amide inhibition, methionine, adenine and histidine may be regarded as end-products of the activity of certain enzyme systems for which $p$-AB is an essential component. The results confirm those of Cutts \& Rainbow (1950), who found that relatively high concentrations of 
methionine, adenine and histidine could replace $p$-AB (albeit not completely) for the growth of Yeast 47. With Yeast 47, the difference in sensitivity to $\mathrm{S}$-amide between the systems involved in the syntheses of methionine, adenine and histidine suggests that three different enzyme systems, each requiring $p$ - $\mathrm{AB}$, are involved. That $p-\mathrm{AB}$ is concerned in the synthesis of an intermediate, common to all three substances, seems less likely.

The inhibitory effect of norleucine in the auxanographs of Yeasts 47 and 70 on media containing no methionine is ascribable to the synergistic effect exercised by norleucine and suphonamides and which may be due to interference by norleucine with methionine metabolism, since the latter two substances are structurally analogous.

Guanine is as effective as adenine in overcoming inhibition by S-amide with Yeasts 47 and 70, but it cannot replace adenine in $p$-AB-free medium for Yeast 47 (Cutts \& Rainbow, 1950). Thus, Yeast 47 can convert adenine to guanine, but not guanine to adenine. Guanine may therefore exert its action as an antisulphonamide by 'sparing' the product of a reaction inhibited by S-amide, viz. adenine. Comparison may be made with the work of Kerr, Seraidarian \& Brown (1951) who found that, whereas isotopically labelled adenine was incorporated into the ribonucleic acid of Torulopsis utilis both as adenine and guanine, labelled guanine appeared only as guanine.

There is no evidence that PGA is an essential cell constituent for the yeasts studied. Other tests with Yeast 47, not reported here, confirm that PGA will neither replace $p$-AB nor raise the level of growth obtainable in the presence of methionine, adenine and histidine in $p$-AB-free medium. The explanation of the inactivity of synthetic PGA for yeasts possibly lies in Woods's (1948) suggestion that synthetic PGA may not be identical with (or convertible to) all natural forms of 'folic acid'.

\section{REFERENCES}

Cutts, N. S. \& Rainbow, C. (1950). Studies of a yeast exacting towards $p$-aminobenzoic acid. J. gen. Microbiol. 4, 150.

HARris, J. S. \& KoHN, H. I. (1941). The effects of purines on the action of sulphonamides. J. biol. Chem. $141,989$.

Emery, W. B., McLeod, N. \& Robinson, F. A. (1946). Comparative microbiological assays of members of the vitamin $B$ complex in yeast and liver extracts. Biochem. J. 40, 426.

Kerr, S. E., Seraidarian, K. \& Brown, G. B. (1951). On the utilization of purines and their ribose derivatives by yeast. J. biol. Chem. 188, 207.

Koft, B. W., Sevag, M. G. \& Steers, E. (1950). The true nature of the stimulation of the growth of Lactobacillus arabinosus $17-5$ by folic acid. J. biol. Chem. $185,9$.

LAMPEN, K. O. \& JonES, M. J. (1947). The growth-promoting and antisulphonamide activity of $p$-aminobenzoic acid, pteroylglutamic acid and related compounds for Lactobacillus arabinosus and Streptobacterium plantarum. J. biol. Chem. 170, 133.

LAMPen, J. O., Jones, M. J. \& Roepke, R. R. (1949). Mutant strains of Escherichia coli unable to synthesise $p$-aminobenzoic acid. J. biol. Chem. 180, 423.

Northam, B. E. \& NorRIs, F. W. (1951). Growth requirements of Schizosaccharomyces octosporus, a yeast exacting towards adenine. J. gen. Microbiol. 5, 502. 
RAINBow, C. (1948). $p$-Aminobenzoic acid a growth factor for certain brewer's yeasts. Nature, Lond., 162, 572.

Shive, W. \& Roberts, E. C. (1946). Biochemical transformations as determined by competitive analogue-metabolite growth inhibitions. Some transformations involving $p$-aminobenzoic acid. J. biol. Chem. 162, 463.

SNell, E. C. \& Mitchell, H. K. (1942). Some sulphanilamide antagonists as growth factors for lactic acid bacteria. Arch. Biochem. $1,93$.

Winkler, K. C. \& HaAN, P. G. de (1948). The action of sulphanilamide. XII. Non-competitive antagonists for E. coli. Arch. Biochem. 18, 743.

Woons, D. D. (1948). Les sulfamides en tant qu'antagonistes de l'acide $p$-aminobenzoïque. Bull. Soc. Chim. biol. 30, 730.

(Received 24 February 1951) 